\title{
Fractional Plasticity Model for Coarse Aggregates Incorporating Particle Breakage under Triaxial Loading
}

\author{
Jiangong Yang $\mathbb{D}^{1}$ and Yifei Sun $\mathbb{D D}^{2}$ \\ ${ }^{1}$ Tianjin College, University of Science and Technology Beijing, Tianjin 301800, China \\ ${ }^{2}$ Faculty of Civil and Environmental Engineering, Ruhr-Universität Bochum, Bochum 44801, Germany \\ Correspondence should be addressed to Yifei Sun; yifei.sun@rub.de
}

Received 19 October 2020; Revised 23 February 2021; Accepted 9 March 2021; Published 22 March 2021

Academic Editor: Yingchun Li

Copyright ( 2021 Jiangong Yang and Yifei Sun. This is an open access article distributed under the Creative Commons Attribution License, which permits unrestricted use, distribution, and reproduction in any medium, provided the original work is properly cited.

\begin{abstract}
Fractional plasticity has been originally proposed for capturing the state-dependent nonassociated behaviour of sand, with no use of state variables and additional plastic potentials. However, for coarse aggregates, significant particle breakage would occur, which could influence the stress-strain behaviour of the material, by shifting the critical state line in the $e-\ln p^{\prime}$ plane. In this note, an extension of the fractional plasticity for constitutive modelling of coarse aggregates is made by incorporating particle breakage under triaxial loading. The developed model is validated by simulating a series of laboratory test results of different coarse aggregates under triaxial loading, where a good agreement between the model simulations and test results is observed.
\end{abstract}

\section{Introduction}

Coarse granular aggregates, e.g., rockfill and ballast, have been widely used in constructing public facilities, due to its high mechanical qualities [1-4]. However, it has been long found that when subjected to external loads, particle breakage would occur in coarse aggregates, which significantly influenced the strength and deformation behaviour of the material [5-9]. For example, reduced volumetric dilatancy usually took place with increasing particle breakage [7]. The Mohr-Coulomb strength decreased as the stress level increased because a significant extent of particle breakage occurred in the material. Most importantly, particle breakage would change the critical state behaviour of coarse aggregates. For example, Yu [10] reported that particle breakage would shift down the critical state line (CSL) of rockfill in the $e-\ln p^{\prime}$ plane, where $e$ and $p^{\prime}$ are the void ratio and mean effective principal stress, respectively. Similar observations have been also reported elsewhere [11]. However, traditional constitutive approaches for soils were mainly based on linear critical state lines, which may not be suitable for coarse granular soils that exhibit high extent of particle breakage. To solve this problem, a series of elastoplastic models incorporating the concept of particle breakage have been proposed $[12,13]$. However, an additional plastic potential was usually required for unified modelling of the nonassosiated stress-strain behaviour of coarse soils. Recently, a novel approach, the fractional plasticity [14-17], has been developed for modelling the state-dependent nonassociated behaviour for geomaterials, where no use of the additional plastic potentials was required. However, this approach did not consider the effect of particle breakage on the constitutive behaviour of highly crushable soils.

Therefore, this study attempts to extend the original fractional plasticity for crushable coarse aggregates, by incorporating the concept of particle breakage. For the sake of simplicity, only homogenous and isotropic coarse aggregates under triaxial loads are considered here. For more complex loading conditions, one can refer to Sun et al. [15].

\section{Fractional Plasticity considering Particle Breakage}

2.1. Plastic Loading Direction. Following previous studies $[14,15]$, the loading surface $(f)$ can be formulated as 


$$
f=\left(\frac{q}{M}\right)^{2}+p^{\prime 2}-p^{\prime} p_{0}^{\prime}=0
$$

where $p_{0}^{\prime}$ denotes the intercept of $f$ with the $p^{\prime}$-axis, $M$ is the critical state stress ratio, $\left[p^{\prime}=\left(\sigma_{3}^{\prime}+2 \sigma_{3}^{\prime}\right) / 3\right]$ and $q$ $\left[=\sigma_{1}^{\prime}-\sigma_{3}^{\prime}\right]$ are the mean effective and deviator stresses, respectively, and $\sigma_{1}^{\prime}$ and $\sigma_{3}^{\prime}$ are the first and third effective principal stresses, respectively. The loading direction (n) can be obtained from the first-order derivatives of $f$, such that

$$
\mathbf{n}=\left[n_{v}, n_{s}\right]^{T}=\left[\frac{M^{2}-\eta^{2}}{\sqrt{4 \eta^{2}+M^{4}-2 M^{2} \eta^{2}+\eta^{4}}}, \frac{2 \eta}{\sqrt{4 \eta^{2}+M^{4}-2 M^{2} \eta^{2}+\eta^{4}}}\right]^{T} .
$$

2.2. Plastic Flow Direction. Instead of using an additional plastic potential, the plastic flow direction (m) in fractional plasticity $[14,15]$ is directly obtained by conducting the fractional derivatives of $f$, such that

$$
\mathbf{m}=\left[m_{v}, m_{s}\right]^{T}=\left[\frac{d_{g}}{\sqrt{1+d_{g}^{2}}}, \frac{1}{\sqrt{1+d_{g}^{2}}}\right]^{T},
$$

where

$$
\begin{aligned}
d_{g} & =\frac{\partial^{\alpha} f / \partial p^{\prime \alpha}}{\partial^{\alpha} f / \partial q^{\alpha}}, \\
& =M^{1+\alpha} \frac{\left(p^{\prime}-p_{c}^{\prime}\right)+(2-\alpha)\left(p_{c}^{\prime}-p_{0}^{\prime} / 2\right)}{\left(q-q_{c}\right)+(2-\alpha) q_{c}},
\end{aligned}
$$

in which $\alpha=\exp \left[k\left(1-p^{\prime} / p_{0}^{\prime}\right)\right]$, is used, considering the initial isotropic stress-state, where $q=0$ and $d_{g} \longrightarrow+\infty$. $k$ is a material constant. Note that the definition of the fractional derivatives can be found in appendix. A dependence of the plastic flow direction on the material state is observed in equation (4), where not only $p^{\prime}$ and $q$ but also $p^{\prime}-p_{c}^{\prime}$ and $q-q_{c}$ determine the value of $d_{g} . q_{c}$ can be calculated from the relative position between the current stress point and CSL in the $p^{\prime}-q$ plane [14], such that

$$
\frac{q_{c}-q}{p^{\prime}-p_{c}^{\prime}}=\frac{q-q_{c}}{p_{c}^{\prime}-p^{\prime}}=M,
$$

while $p_{c}^{\prime}$ can be obtained from the CSL in the $e-\ln p^{\prime}$ plane. As shown in many studies $[7,10]$, particle breakage would shift down the CSL. Therefore, a CSL with evolving intercept should be used for constitutive modelling. Considering the fact that both strain variation [18] and stress variation [2] could induce significant particle breakage, an energy-based CSL suggested by Liu and Zou [11] is used in this study for determining $p_{c}^{\prime}$ :

$$
p_{c}^{\prime}=p_{r} \exp \left[\left(e_{\Gamma 0}-\frac{e_{\Gamma 0} w_{p}}{a w_{p}+b}-e\right) / \lambda\right],
$$

where $p_{r}=1 \mathrm{kPa}$ is the unit pressure, $\lambda$ is the gradient of the CSL in the $e-\ln p^{\prime}$ plane, $e$ is the current void ratio, $w_{p}=$ $\int \sigma_{i j}^{\prime} d \varepsilon_{i j}$ is the plastic energy density, $\sigma_{i j}^{\prime}$ and $\varepsilon_{i j}$ are the stress and strain tensors, respectively, and $e_{\Gamma 0}, a$, and $b$ are material constants. Through equation (6), the effect of particle breakage on the stress-strain behaviour of coarse aggregates can be thus considered.
2.3. Hardening Modulus. The plastic modulus, $H$, can be obtained by using the consistency condition together with the evolving law, i.e., $\Delta h=\langle L\rangle \bar{h}$, for the hardening parameter $(h)$. Here, $\Delta$ indicates increment. Hence,

$$
H=-\frac{\partial f}{\partial h} \bar{h},
$$

where $L=\mathbf{m}^{T} \Delta \sigma^{\prime} / H$ and $\bar{h}$ can be defined by the law for deviatoric hardening. During loading, $f$ would evolve until reaching the critical state, where the evolution should be bounded by the peak stress surface. Therefore, one can write

$$
\bar{h}=\frac{\chi G\left(M_{p}-\eta\right)}{q},
$$

where $G=G_{0}(2.97-e)^{2} /(1+e) \sqrt{p^{\prime} p_{a}}$ is the shear modulus [14]. $G_{0}$ is a material constant. $p_{a}=100 \mathrm{kPa}$ is the atmospheric pressure. $\chi$ is a model parameter and can be a function of the void ratio, e.g., $\chi=\chi_{1}(1-e)$, for better model performance. $\chi_{1}$ is a model constant. $M_{p}=M[1+$ $\left.\beta\left(\sqrt{p_{c} / p^{\prime}}-1\right)\right]$ is the peak stress ratio [19], in which $\beta$ is a material constant. Further substituting equations (1) and (8) into equation (7), $H$ can be obtained as

$$
H=\chi G\left(\frac{M_{p}}{\eta}-1\right) .
$$

In addition to the plastic strain, coarse aggregates may also experience elastic strain during loading, which can be described via Hooke's elastic law. Accordingly, the elastoplastic relation for coarse aggregates can be provided as

$$
\left\{\begin{array}{l}
\Delta \varepsilon_{v}=\frac{\Delta p^{\prime}}{K}+\left(\frac{n_{v}}{H} \Delta p^{\prime}+\frac{n_{s}}{H} \Delta q\right) m_{v}, \\
\Delta \varepsilon_{s}=\frac{\Delta q}{3 G}+\left(\frac{n_{v}}{H} \Delta p^{\prime}+\frac{n_{s}}{H} \Delta q\right) m_{s},
\end{array}\right.
$$

where the bulk modulus $K=(((2+2 v) G / 3) /(1-2 v))$ and $v$ is Poisson's ratio.

\section{Parameter Identification and Model Validation}

3.1. Parameter Identification. There are ten parameters $\left(G_{0}\right.$, $\nu, M, \lambda, e_{\Gamma 0}, a, b, k, \beta$, and $\left.\chi_{1}\right)$ in the extended fractional plasticity model, which can be determined via triaxial tests. 
TABLE 1: Model parameters.

\begin{tabular}{|c|c|c|c|c|c|c|c|c|c|c|}
\hline Material & $G_{0}$ & $v$ & $M$ & $\lambda$ & $e_{\Gamma 0}$ & $a$ & $b$ & $\beta$ & $k$ & $\chi_{1}$ \\
\hline Shah Nehar rockfill [2] & 20 & 0.35 & 1.4 & 0.04 & 0.56 & 60 & 120 & 0.05 & 0.08 & 6 \\
\hline Purulia dam rockfill [2] & 40 & 0.35 & 1.46 & 0.03 & 0.53 & 12 & 75 & 0.2 & 0.05 & 1.5 \\
\hline Railroad ballast [5] & 80 & 0.20 & 1.9 & 0.23 & 2.1 & 10 & 6000 & 0.12 & 0.2 & 1.2 \\
\hline Limestone ballast [20] & 90 & 0.25 & 1.75 & 0.078 & 1.18 & 150 & 450 & 0.23 & 0.05 & 2 \\
\hline Crushed basalt [21] & 73 & 0.25 & 2.04 & 0.19 & 1.83 & 10 & 5000 & 0.45 & 0.1 & 1.8 \\
\hline
\end{tabular}

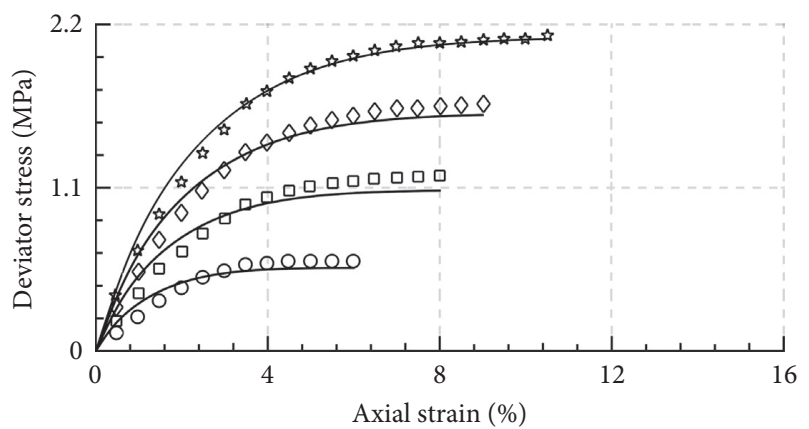
○ $\sigma_{3}^{\prime}=0.2 \mathrm{MPa}$
$\sigma_{3}^{\prime}=0.4 \mathrm{MPa}$
$\diamond \sigma_{3}^{\prime}=0.6 \mathrm{MPa}$
מ $\sigma_{3}^{\prime}=0.8 \mathrm{MPa}$
Prediction

(a)

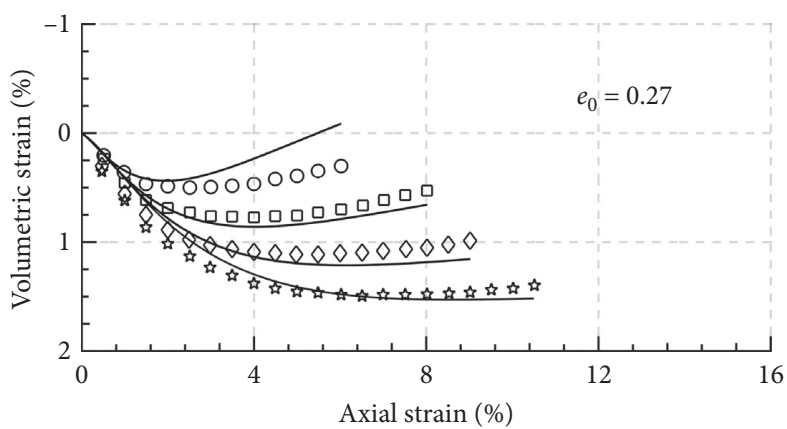

(b)

FIGURE 1: Model simulations of the test results of Shah Nehar rockfill [2].

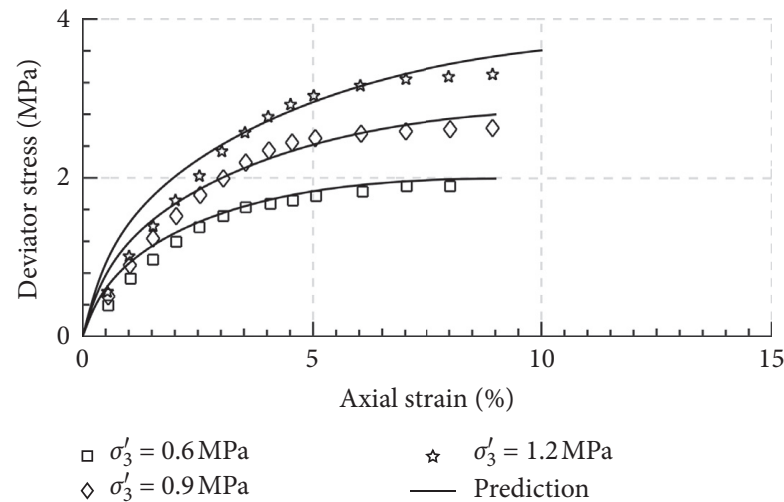

(a)

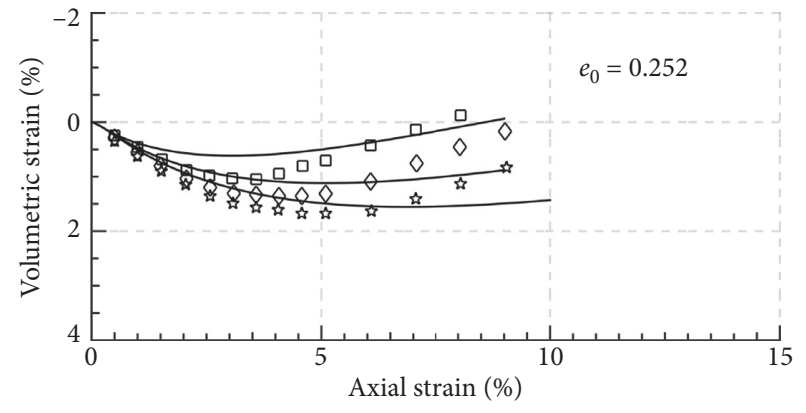

(b)

Figure 2: Model simulations of the test results of Purulia dam rockfill [2].

$G_{0}$ and $v$ can be calculated from the $\varepsilon_{s}-q$ curve at the initial loading stage, where $G_{0} \approx(1+e) G /(2.97-e)^{2} /\left(p^{\prime} p_{a}\right)^{0.5}$ and $\nu \approx\left(3 \varepsilon_{s}-2 \varepsilon_{v}\right) /\left(6 \varepsilon_{s}+2 \varepsilon_{v}\right)$. $M$ is determined by measuring the gradient of the CSL in the $p^{\prime}-q$ plane. Another four critical state parameters, $\lambda, e_{\Gamma 0}, a$, and $b$, can be determined by fitting the critical state points in the $e-\ln p^{\prime}$ plane, as comprehensively discussed in Liu and Zou [11]. $k$ can be determined from the phase transformation state of the material $\left(d_{g}=0\right)$, where

$$
k=\frac{p_{0 t}^{\prime}}{p_{0 t}^{\prime}-p_{t}^{\prime}} \ln \frac{2 M^{2} p_{c t}^{\prime}-2 \eta_{t}^{2} p_{t}^{\prime}}{2 M^{2} p_{c t}^{\prime}-M^{2} p_{t}^{\prime}-\eta_{t}^{2} p_{t}^{\prime}},
$$

where $e_{t}, p_{t}^{\prime}, p_{c t}^{\prime}, p_{0 t}^{\prime}$, and $\eta_{t}$ are the void ratio, mean effective stress, critical mean stress, intercept of $f$ with $p^{\prime}$-axis, and stress ratio, respectively, at the phase transformation state. Moreover, $\beta$ can be determined from the peak stress point of coarse aggregates under drained loading $(H=0)$, such that

$$
\beta=\frac{\eta_{p} / M-1}{\sqrt{p_{c p}^{\prime} / p_{p}^{\prime}}-1},
$$

where $e_{p}, p_{p}^{\prime}, p_{c p}^{\prime}$, and $\eta_{p}$ are the void ratio, mean effective stress, critical mean stress, and stress ratio, respectively, at the peak state. In addition, $\chi_{1}$ can be obtained by fitting the 


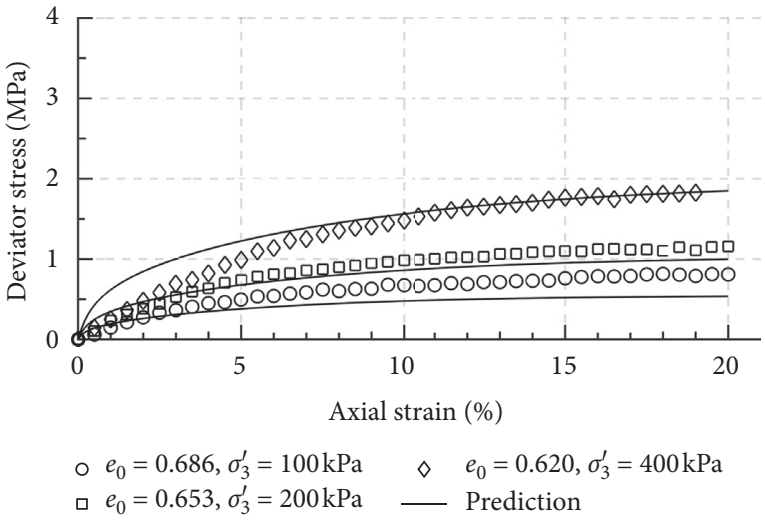

(a)

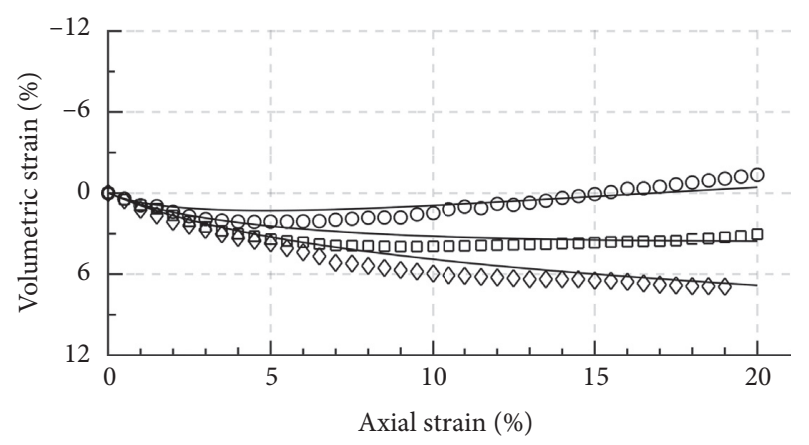

(b)

Figure 3: Model simulations of the test results of dense ballast [5].

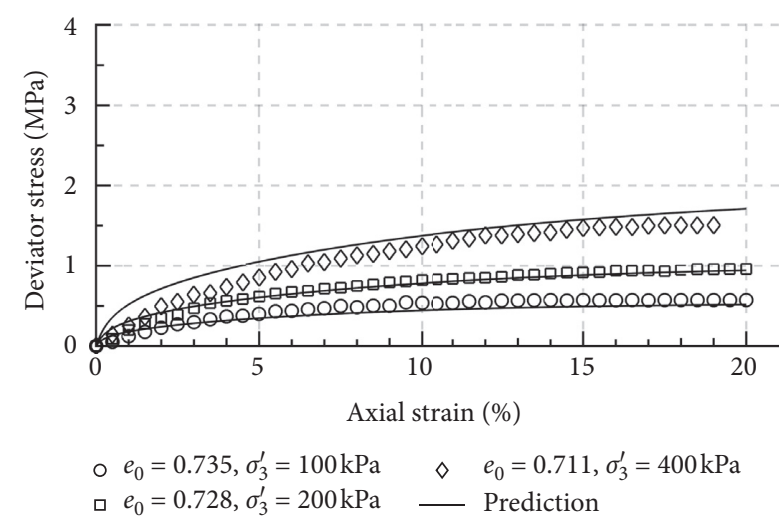

(a)

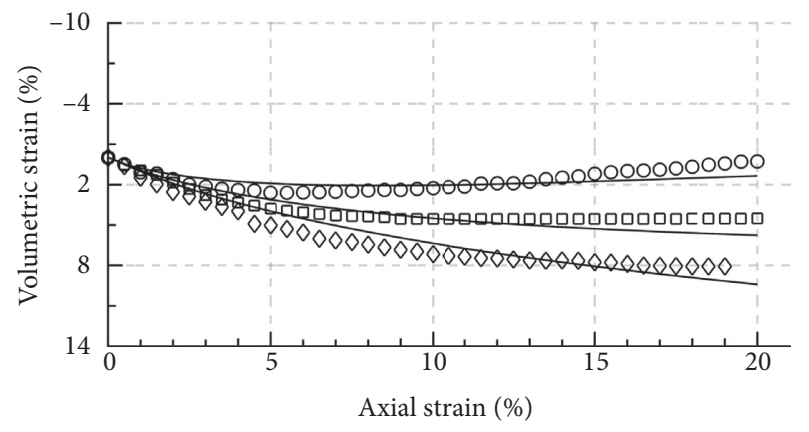

(b)

Figure 4: Model simulations of the test results of medium dense ballast [5].

$\varepsilon_{1}-q$ relationship of samples with different $e_{0}$ [14]. The values of each model parameter are listed in Table 1.

3.2. Model Validation. To validate the extend fractional plasticity approach, a series of triaxial test results of different coarse aggregates are simulated and discussed. Details of the material properties and test setup can be found in the relevant literatures $[2,5,20,21]$ and thus are not introduced here for the sake of simplicity. The model simulations of the stress-strain behaviour of coarse aggregates, e.g., rockfill [2] and ballast $[5,20,21]$, are represented in Figures 1-6.

Figure 1 shows the model predictions of the test results of Shah Nehar rockfill [2]. $e_{0}$ of 0.27 with $\sigma_{3}^{\prime}$ of $200-800 \mathrm{kPa}$ was used for model implementation. It can be found that the extended fractional plasticity model can well describe the typical stress-strain response, including volumetric contraction/dilation and strain hardening/softening, of Shah Nehar rockfill. Figure 2 presents the model simulations of the stress-strain behaviour of Purulia dam rockfill [2]. It was reported that the material mainly consisted of angular/ subangular metamorphic rock particles. $e_{0}$ of 0.252 with $\sigma_{3}^{\prime}$ of 600-1200 kPa was used for model implementation. It can be also found from Figure 2 that the model can reasonably capture the stress-strain behaviour of Purulia dam rockfill under different loading pressures.

Figures 3-4 simulate the test results of ballast with two different relative densities [5]. $e_{0}=0.620-0.686$ (dense ballast) and $e_{0}=0.711-0.735$ (medium dense ballast) with $\sigma_{3}^{\prime}$ of $100-400 \mathrm{kPa}$ were used for model simulation. It is found that the extended model can provide reasonable predictions of the stress-strain behaviour of ballast with different relative densities. However, a slight underestimation of the peak stress under low $\sigma_{3}^{\prime}$ is observed. This can be attributed to the low dilatancy predicted by the developed model. A higher extent of dilatancy would result in better model simulations. Figure 5 shows the model simulations of the triaxial behaviour of limestone ballast [20]. $e_{0}=0.721-0.683$ with $\sigma_{3}^{\prime}$ of $10-60 \mathrm{kPa}$ were used for model simulation. An overestimation of the volumetric dilation of limestone ballast can be found in Figure 5. However, the overall predictions of the stress-strain relations are satisfactory. Figure 6 shows the model predictions of stress-strain behaviour of crushed basalt [21]. $e_{0}=0.627-0.696$ with $\sigma_{3}^{\prime}$ of $50-300 \mathrm{kPa}$ was used 


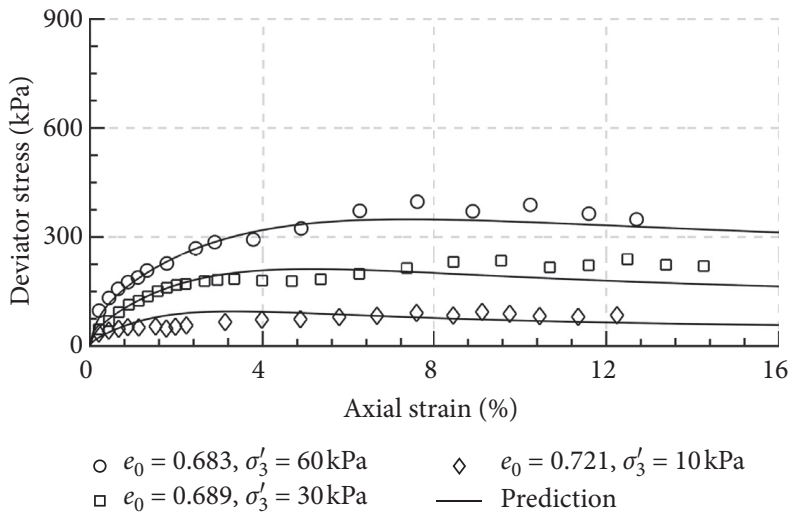

(a)

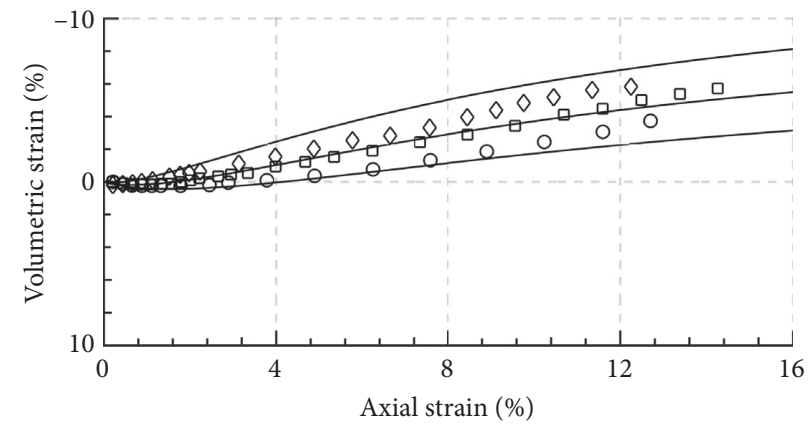

(b)

FIGURE 5: Model simulations of the test results of limestone ballast [20].
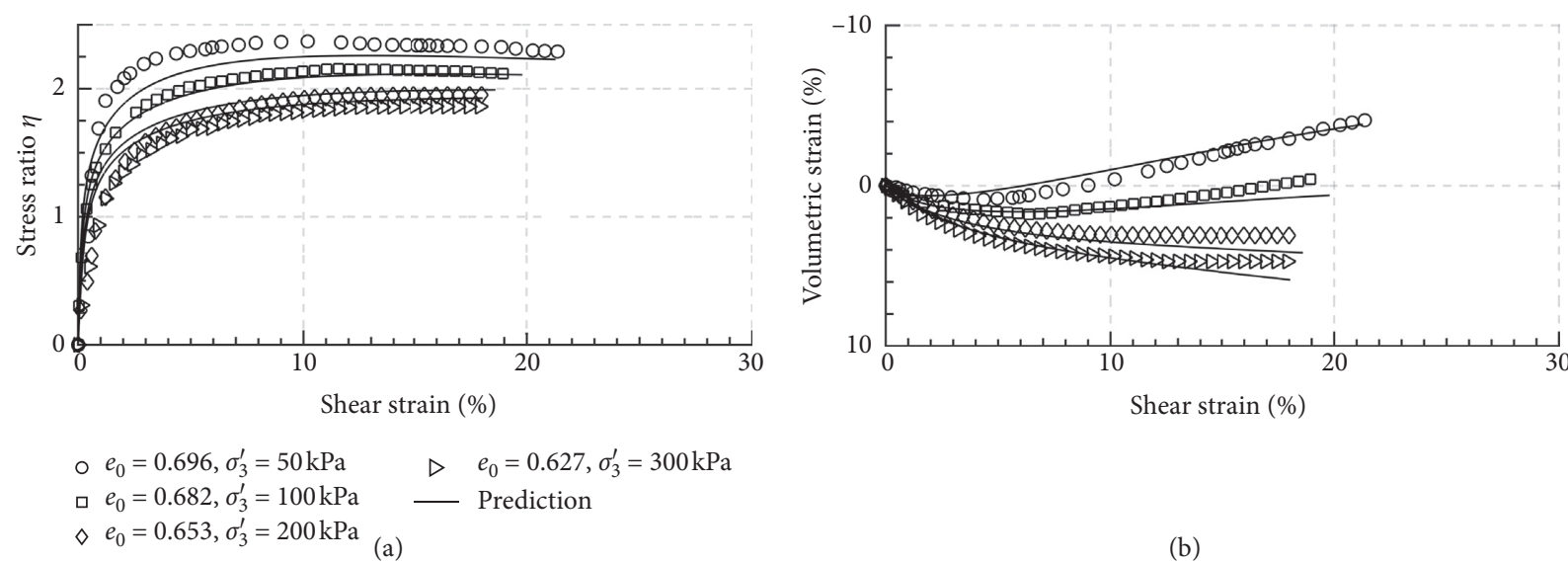

Figure 6: Model simulations of the test results of crushed basalt [21].

for model predictions. It can be found that the proposed model simulates well the relationship between volumetric strain and shear strain for crushed basalt. An underestimation of the peak stress under low $\sigma_{3}^{\prime}$ is also observed, but the overall evolution trend of the deviator stress with the shear strain is captured.

\section{Conclusions}

This study enriched the original fractional plasticity by incorporating particle breakage. Several main findings are summarised as follows:

(1) A state-dependent fractional plasticity was developed by considering the effect of particle breakage on the critical state behaviour of coarse aggregates. The loading rule and plastic flow rule were obtained by conducting first-order derivative and fractional-order derivative at the yielding surface, respectively, while the state-dependent hardening modulus was developed from consistency condition.
(2) The proposed model had ten parameters which can be determined from laboratory tests. Through simulating a series of test results of different coarse aggregates, it was found that the model can reasonably capture the stress-strain behaviour of coarse aggregates with particle breakage.

\section{Appendix}

Caputo's fractional derivatives are used in this study [14]:

$$
\begin{aligned}
D a \sigma^{\alpha} f\left(\sigma^{\prime}\right) & =\frac{1}{\Gamma(n-\alpha)} \int_{a}^{\sigma^{\prime}} \frac{f^{n}(\chi) d \chi}{\left(\sigma^{\prime}-\chi\right)^{\alpha+1-n}}, \quad \sigma^{\prime}>a, \\
D_{\sigma a}^{\prime \alpha} f\left(\sigma^{\prime}\right) & =\frac{(-1)^{n}}{\Gamma(n-\alpha)} \int_{\sigma^{\prime}}^{a} \frac{f^{n}(\chi) d \chi}{\left(\chi-\sigma^{\prime}\right)^{\alpha+1-n}}, \quad a>\sigma^{\prime},
\end{aligned}
$$

where $D$ denotes partial differentiation, $\alpha$ is the fractional order, $a$ and $\sigma^{\prime}$ are the integral limits, $\sigma^{\prime}$ denotes the effective 
stress, in this study, $n$ is a positive integer, and $f$ is the yielding/loading surface function.

\section{Data Availability}

The data used to support the findings of this study are included within the article.

\section{Conflicts of Interest}

The authors declare that they have no conflicts of interest.

\section{Acknowledgments}

The financial support provided by the Scientific Research Project of Tianjin Education Commission (2019KJ145) is appreciated. We would also like to thank Prof. Wen Chen for his inspiration.

\section{References}

[1] S. Nimbalkar, V. S. R. Annapareddy, and A. Pain, "A simplified approach to assess seismic stability of tailings dams," Journal of Rock Mechanics and Geotechnical Engineering, vol. 10, no. 6, pp. 1082-1090, 2018.

[2] A. Varadarajan, K. G. Sharma, S. M. Abbas, and A. K. Dhawan, "Constitutive model for rockfill materials and determination of material constants," International Journal of Geomechanics, vol. 6, no. 4, pp. 226-237, 2006.

[3] Y. Tang, T. Vo, A. Taiebat Hossein, and R. Russell Adrian, "Influences of suction on plate load tests on unsaturated silty sands," Journal of Geotechnical and Geoenvironmental Engineering, vol. 144, no. 8, Article ID 04018043, 2018.

[4] Y. Tang, A. Taiebat Hossein, and R. Russell Adrian, "Numerical modeling of consolidation of unsaturated soils considering hydraulic hysteresis," International Journal of Geomechanics, vol. 18, no. 2, Article ID 04017136, 2018.

[5] Y. Sun, B. Indraratna, J. P. Carter, T. Marchant, and S. Nimbalkar, "Application of fractional calculus in modelling ballast deformation under cyclic loading," Computers and Geotechnics, vol. 82, pp. 16-30, 2017.

[6] M. Liu, Y. Gao, and H. Liu, "An elastoplastic constitutive model for rockfills incorporating energy dissipation of nonlinear friction and particle breakage," International Journal for Numerical and Analytical Methods in Geomechanics, vol. 38, no. 9, pp. 935-960, 2013.

[7] Y. Xiao, H. Liu, Q. Chen, L. Long, and J. Xiang, "Evolution of particle breakage and volumetric deformation of binary granular soils under impact load," Granular Matter, vol. 19, no. 4 , p. 71, 2017.

[8] V. Bandini and M. R. Coop, "The influence of particle breakage on the location of the critical state line of sands," Soils and Foundations, vol. 51, no. 4, pp. 591-600, 2011.

[9] R. P. Singh, S. Nimbalkar, S. Singh, and D. Choudhury, "Field assessment of railway ballast degradation and mitigation using geotextile," Geotextiles and Geomembranes, vol. 48, no. 3, p. 275, 2020.

[10] F. Yu, "Particle breakage and the undrained shear behavior of sands," International Journal of Geomechanics, vol. 18, no. 7, Article ID 04018079, 2018.

[11] H. Liu and D. Zou, "Associated generalized plasticity framework for modeling gravelly soils considering particle breakage," Journal of Engineering Mechanics, vol. 139, no. 5, pp. 606-615, 2013.

[12] Y. Xiao, H. Liu, Y. Chen, J. Jiang, and W. Zhang, "Statedependent constitutive model for rockfill materials," International Journal of Geomechanics, vol. 15, no. 5, Article ID 04014075, 2014.

[13] I. Einav, "Breakage mechanics-Part II: modelling granular materials," Journal of the Mechanics and Physics of Solids, vol. 55, no. 6, pp. 1298-1320, 2007.

[14] Y. Sun, Y. Gao, and Q. Zhu, "Fractional order plasticity modelling of state-dependent behaviour of granular soils without using plastic potential," International Journal of Plasticity, vol. 102, pp. 53-69, 2018.

[15] Y. Sun, Y. Gao, S. Song, and C. Chen, “Three-dimensional state-dependent fractional plasticity model for soils," International Journal of Geomechanics, vol. 19, 2019.

[16] D. Lu, J. Liang, X. Du, C. Ma, and Z. Gao, "Fractional elastoplastic constitutive model for soils based on a novel 3D fractional plastic flow rule," Computers and Geotechnics, vol. 105, pp. 277-290, 2019.

[17] D. Lu, X. Zhou, X. Du, and G. Wang, "A 3D fractional elastoplastic constitutive model for concrete material," International Journal of Solids and Structures, vol. 165, p. 160, 2019.

[18] M. R. Coop, K. K. Sorensen, T. Bodas Freitas, and G. Georgoutsos, "Particle breakage during shearing of a carbonate sand," Géotechnique, vol. 54, no. 3, pp. 157-163, 2004.

[19] Z.-L. Wang, Y. F. Dafalias, X.-S. Li, and F. I. Makdisi, "State pressure index for modeling sand behavior," Journal of Geotechnical and Geoenvironmental Engineering, vol. 128, no. 6, pp. 511-519, 2002.

[20] B. Aursudkij, G. R. McDowell, and A. C. Collop, "Cyclic loading of railway ballast under triaxial conditions and in a railway test facility," Granular Matter, vol. 11, no. 6, pp. 391-401, 2009.

[21] W. Salim and B. Indraratna, "A new elastoplastic constitutive model for coarse granular aggregates incorporating particle breakage," Canadian Geotechnical Journal, vol. 41, no. 4, pp. 657-671, 2004. 